\title{
Coefficients de diversité des populations dans l'écosystème souterrain
}

\author{
R.R. TERCAFS *
}

INTRODUCTION

Dans les pays tempérés froids, l'écosystème souterrain est le siège d'importants mouvements de population. Annuellement, il se produit un double transfert d'animaux. En été, des Trogloxènes pénètrent dans les cavernes pour estiver. En automne, d'autres espèces de Trogloxènes viennent hiberner. Ces phénomènes commencent à être bien connus sur le plan qualitatif (Motas et al., 1967) mais ont été peu étudiés quantitativement (Ives, 1964).

En vue de préciser ces phénomènes, nous avons étudié la composition faunique d'une cavité souterraine en considérant les relations entre les deux régions écologiques principales - la zone extérieure et la zone profonde - et leur évolution en fonction du temps. Pour exprimer ces relations, nous avons utilisé les équations classiques de la théorie de l'information appliquées à l'étude des coefficients de similarité des communautés (Pielou, 1966, 1967; Horn, 1966).

\section{MATERIEL ET METHODES}

La caverne choisie ('Trou des Nutons', Chaudfontaine, Belgique) possède une géométrie relativement simple. Elle est constituée d'un couloir horizontal d'une vingtaine de mètres de long (figure 1). Le porche d'entrée est fortement soumis aux conditions climatiques extérieures et ne contient pratiquement pas d'animaux. Aussi a-t-il été négligé dans cette étude. Les observations portent uniquement sur les animaux terrestres. La méthode des pièges n'a pas été utilisée car, dans une grotte de petites dimensions, elle peut apporter des modifications considérables dans la répartition faunique (Jequier, 1964). Les relevés ont donc été effectués à vue. A cet effet, la caverne est divisée en quadrats d'un mètre carré, numérotés depuis l'entrée. On obtient ainsi 17 points d'observation où tous les animaux observés sur les parois et sur le sol sont comptabilisés, espèce par espèce. La caverne choisie contient les principales espèces rencontrées dans des cavernes de situation géographique analogue. La liste des espèces rencontrées est présentée dans le tableau 1. Les observations sont effectuées, pour la période estivale, le 10 juin 1971 et le 17 novembre 1971 pour la période automnale.

\footnotetext{
* Chercheur qualifié du Fonds National Belge de la Recherche Scientifique.
} Institut Royal des Sciences Naturelles, 31 rue Vautier, Bruxelles, Belgique 


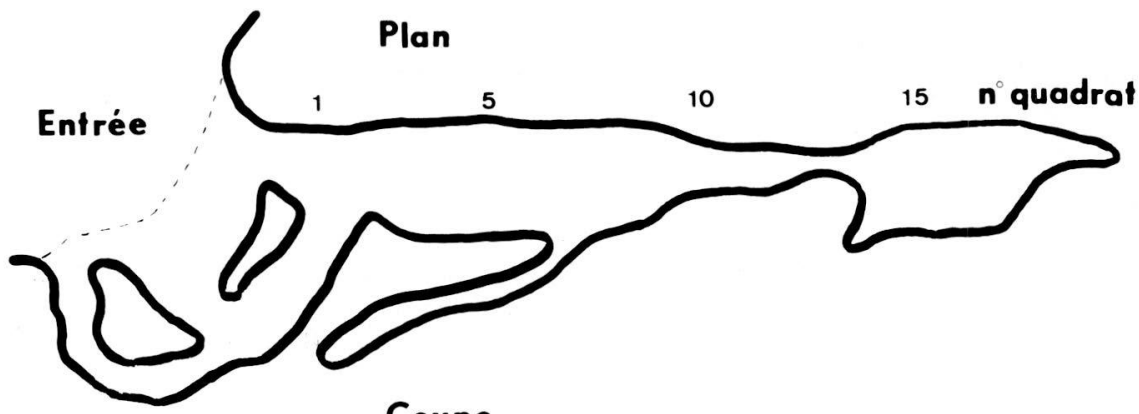

\section{Coupe}

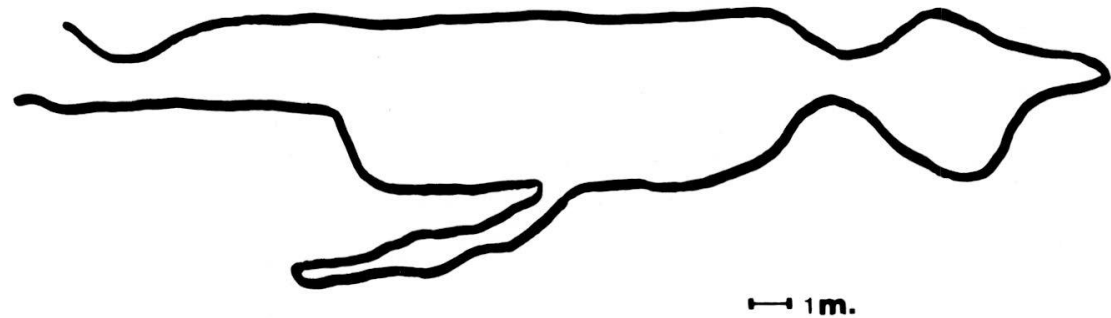

\section{Trou des Nutons}

\section{Chaudfontaine}

Fig. 1. Plan et coupe de la caverne 'Trou des Nutons' à Chaudfontaine, Belgique. Positions des quadrats.

Tableau 1. Liste des espèces observées.

Mollusques.

Zonitidae:

Oxychilus cellarius Müll.

Arachnides.

Argiopidae:

Agenelidae:

Erigonidae:

Meta menardi Latr.

Tegeneria silvestris Clerck.

Porrhoma sp.

Myriapodes.

Blaniulidae:

Blaniulus guttulatus Bosc.

Insectes.

Lépidoptères.

Geometridae:

Noctuidae:

Diptères.

Culicidae:

Scoliopteryx libatrix L.

Mycetophilidae: Speolepta leptogaster Winn.

Limoniidae: Limonia nubeculosa $(\mathrm{Mg})$

Trichoptères.

Limnophilidae: Stenophylax permistus Mc. L. 
Pour les calculs des coefficients de diversité, les équations suivantes ont été utilisées:

1. Pour chaque quadrat, pour chacune des 2 périodes (Brillouin, 1960; Piélou, 1966, 1967).

a) Coefficient de diversité observé (HOBS):

$$
\text { HOBS }=\frac{1}{N} \log \frac{N !}{N_{1} ! N_{2} ! \ldots N_{i} !}
$$

où $\mathrm{N}=$ nombre total d'individus

$\mathrm{i}=$ nombre d'espèces

$\mathrm{N}_{\mathbf{i}}=$ nombre d'individus de chaque espèce $\mathrm{i}$

b) Coefficient de diversité maximum (HMAX):

$$
\operatorname{HMAX}=\frac{1}{\mathrm{~N}} \log \frac{\mathrm{N} !}{\left(\left[\frac{\mathrm{N}}{\mathrm{i}}\right] !\right)^{\mathrm{i}-\mathrm{r}}\left(\left[\frac{\mathrm{N}}{\mathrm{i}}\right]+1\right) \stackrel{\mathrm{r}}{!}}
$$

où $\mathrm{N}=$ nombre total d'individus

$\mathrm{i}=$ nombre d'espèces

$\left[\frac{\mathrm{N}}{\mathrm{i}}\right]=$ partie entière $\mathrm{de} \frac{\mathrm{N}}{\mathrm{i}}$

$\mathrm{r}=\mathrm{N}-\mathrm{i}[\mathrm{N} / \mathrm{i}]$

c) Coefficient d'uniformité (COEFJ):

$$
\text { COEFJ }=\frac{\text { HOBS }}{\text { HMAX }}
$$

\section{Dans chacune des populations considérées 2 à 2 .}

Au point de vue écologique, la caverne étudiée peut se subdiviser en deux zones. Une zone extérieure soumise, suivant certaines relations qui seront étudiées dans un travail séparé, aux conditions climatiques extérieures. Une zone profonde où les conditions climatiques sont beaucoup plus stables. Les quadrats 1 à 11 inclus font partie de la zone extérieure. Les comparaisons vont porter sur les populations occupant ces deux zones. 
a) Coefficient de diversité observée (HOBS).

$$
\text { HOBS }=\frac{1}{\mathrm{~N}} \log \frac{\mathrm{N} !}{\mathrm{N}_{1} ! \mathrm{N}_{2} ! \ldots \mathrm{N}_{\mathrm{i}} !}
$$

où $\mathrm{N}=$ nombre total d'individus

$\mathrm{N}_{\mathrm{i}}=$ nombre d'individus de l'espèce i pour les 2 populations

b) Coefficient de diversité maximum (HMAX).

$$
\operatorname{HMAX}=\frac{1}{\mathrm{~N}} \log \frac{\mathrm{N} !}{\mathrm{N}_{1}^{\prime} ! \mathrm{N}_{1}^{\prime \prime} \ldots \mathrm{N}_{1}^{\prime} ! \mathrm{N}_{\mathrm{i}}^{\prime \prime} !}
$$

où $\mathrm{N}=$ nombre total d'individus

$\mathrm{N}_{\mathrm{i}}^{\prime}=$ nombre d'individus de l'espèce $\mathrm{i}$ dans la population 1

$\mathrm{N}_{\mathrm{i}}^{\prime}=$ nombre d'individus de l'espèce $\mathbf{i}$ dans la population 2

c) Coefficient de diversité minimum (HMIN).

$$
\text { HMIN }=\frac{1}{N} \log \frac{N !}{\left(N_{1}^{\max }+N_{2}^{\max }\right) ! \ldots\left(N_{i}^{\max }+N_{j}^{\max }\right) !}
$$

où $\mathrm{N}=$ nombre total d'individus

$\mathrm{N}_{\mathrm{i}}^{\max }=$ nombre d'individus de l'espèce la plus nombreuse en individus

$\mathrm{N}_{\mathrm{j}}^{\max }=$ nombre d'individus de l'espèce de rang inférieur

d) Coefficient de similarité (C).

$$
\mathrm{C}=\frac{\text { HMAX }- \text { HOBS }}{\text { HMAX }- \text { HMIN }}
$$

Les comparaisons suivantes ont été effectuées:

- comparaison entre la zone extérieure et la zone profonde au moment de la saison

1 (été).

- comparaison entre ces deux zones au moment de la saison 2 (automne).

- comparaison entre les zones extérieures à deux saisons différentes.

- comparaison entre les zones profondes à deux saisons différentes. 
La première comparaison a pour but d'étudier la composition faunique globale des deux zones écologiquement différentes de la caverne au moment où le nombre de Trogloxènes est le plus faible (été). La deuxième comparaison s'effectue sur les mêmes zones mais après la période de grand bouleversement du nombres d'espèces lors de la rentrée des Trogloxènes hibernants.

La troisième et la quatrième comparaison portent sur l'évolution d'une même zone écologique (zone extérieure et zone profonde considérées séparément) en considérant deux saisons différentes.

L'ensemble des calculs à effectuer a été programmé en Fortran IV. Les travaux sont confiés à un ordinateur IBM $360 / 65$ couplé en multiprogrammation à un $360 / 44^{*}$.

Tableau 2. - Coefficients de diversité des populations d'un écosystème souterrain. Comparaison entre deux saisons. Saison $1=$ été; saison $2=$ automne. Les quadrats sont numérotés depuis l'entrée de la caverne. HOBS = coefficient de diversité observé. HMAX = coefficient de diversité maximum; COEFJ = coefficient d'uniformité. Les valeurs sont calculées par ordinateur et données en format E14.7. La valeur est donc à multiplier par $10 \mathrm{w}$, w étant le nombre après le signe $\mathrm{E}$.

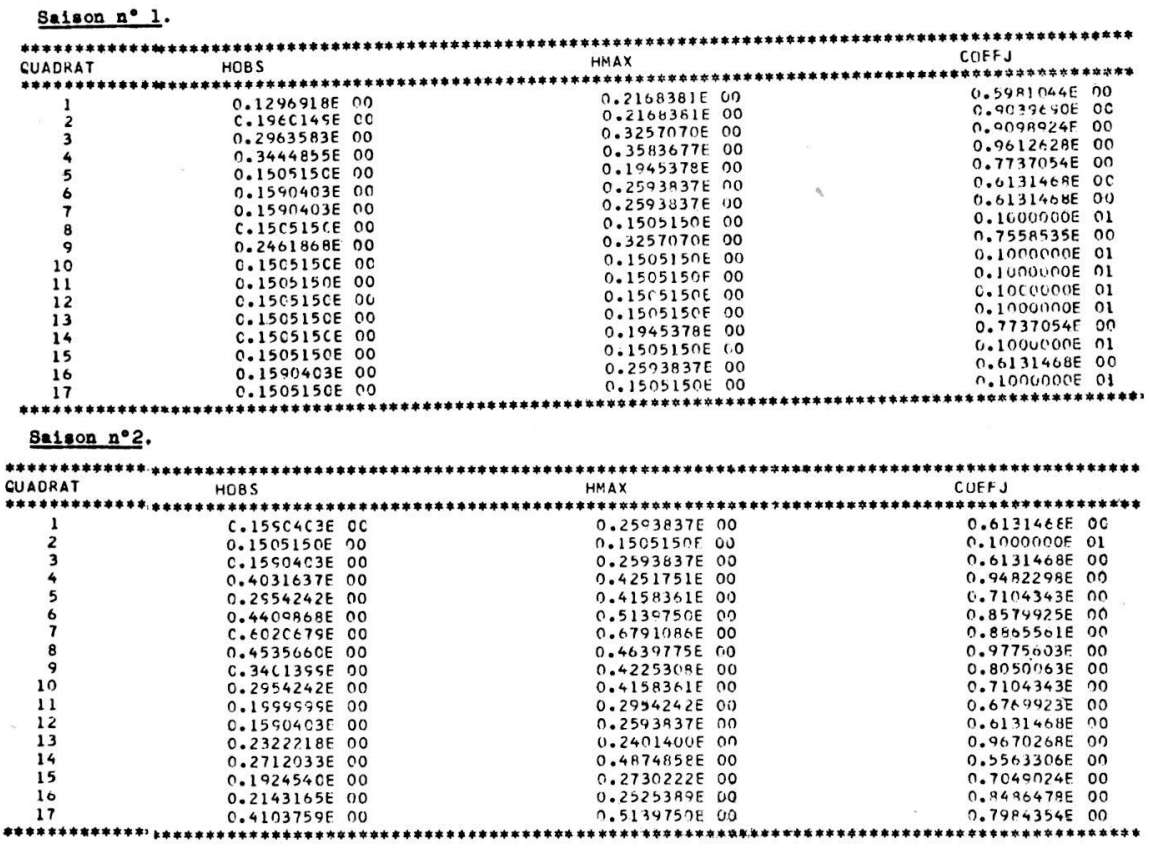

* Passages en machine effectués au Centre de Calcul de l'Université de Liège (Belgique). 


\section{RESULTATS}

1. Coefficients de diversité pour chacun des quadrats.

Les calculs effectués sur chacun des quadrats pendant la saison 1 (été) et la saison 2 (automne) sont présentés dans la tableau 2.

\section{Comparaison entre les zones écologiquement différentes.}

Les résultats obtenus sont exposés dans le tableau 3. Les coefficients de diversité observés, minimum et maximum ainsi que les coefficients de similarité ont été calculés pour chacun des comparaisons envisagées.

Tableau 3. - Coefficients de diversité et de similarité dans un écosystème souterrain. Comparaison entre deux zones écologiquement différentes.

Coefficients de diversité observés.

$\begin{array}{llll}\text { HOBXEP } & \text { HOBYEP } & \text { HOBXYE } & \text { HOBXYP } \\ 0.047 & 0.188 & 0.130 & 0.504\end{array}$

Coefficients de diversité maximum.

$\begin{array}{llll}\text { HAXEP } & \text { HAYEP } & \text { HAXYE } & \text { HAXYP } \\ 0.096 & 0.315 & 0.150 & 0.758\end{array}$

Coefficients de diversité minimum.

$\begin{array}{llll}\text { HMIXEP } & \text { HMIYEP } & \text { HMIXYE } & \text { HMIXYP } \\ 0.005 & 0.020 & 0.019 & 0.012\end{array}$

Coefficients de similarité.

$\begin{array}{llll}\text { CXEP } & \text { CYEP } & \text { CEXY } & \text { CPXY } \\ 0.54 & 0.43 & 0.064 & 0.34\end{array}$

Les abréviations suivantes sont utilisées:

$\mathrm{Y}$ désigne la saison 1 .

$\mathrm{X}$ désigne la saison 2 .

E désigne la zone extérieure.

$P \quad$ désigne la zone profonde.

HOB désigne le coefficient de diversité observé.

HA désigne le coefficient de diversité maximum.

HM I désigne le coefficient de diversité minimum.

C désigne le coefficient de similarité.

Ainsi, par exemple, CXEP correspond à la comparaison entre la zone extérieure et profonde pendant la saison 2 .

\section{DISCUSSION ET CONCLUSION}

Plusieurs hypothèses peuvent être émises pour expliquer les variations de populations dans un écosystème. On peut ainsi distinguer:

- l'influence des facteurs physiques: si un habitat subit des variations climatiques 
saisonnières, il peut s'ensuivre des modifications dans la répartition des espèces au sein de cet habitat en fonction de certaines contraintes physiologiques propres aux espèces considérées.

- l'influence de la productivité: des variations dans la disponibilité de nourriture influencent évidemment les valeurs numériques des populations ainsi que leur distribution spatiale.

- l'influence de la compétition et de la prédation: en relation avec des variations de productivité de l'écosystème, les relations entre les divers niveaux trophiques vont influencer également la composition faunique des divers habitats.

Sur le plan général, ces influences respectives ont été étudiées par divers auteurs (Mac Arthur, 1965; Margalef, 1963) et, pour les populations de cavernicoles, par Poulson et al. (1969) et par Culver et al. (1970). Ces derniers ont ainsi montré une intéressante corrélation entre la diversité des espèces et la diversité du substrat.

Dans le cas de l'écosystème souterrain que nous avons étudié, c'est-à-dire dans une caverne de géométrie simple peuplée principalement de Trogloxènes et de Troglophiles, on devait s'attendre aux résultats suivants:

- la zone profonde aux conditions climatiques stables devrait conserver, en fonction du temps, une certaine stabilité dans sa composition faunique.

- la zone extérieure, par contre, devrait avoir une stabilité faible vu qu'elle renferme la majeure partie des Trogloxènes saisonniers.

C'est bien en effet ce que l'on observe (voir tableau 4: CPXY et CEXY).

- la zone extérieure comparée à la zone profonde au même moment doit posséder un coefficient de similarité élevé car sur l'ensemble de la faune cavernicole, l'apport des Trogloxènes est quantitativement faible. Les résultats obtenus sont en bonne concordance avec ces prévisions puisqu'on obtient $\mathrm{CXEP}=0.54$ et $\mathrm{CYEP}=0.43$. De la même façon l'analyse par quadrat (tableau 2) montre que les coefficients de diversité observé subissent, dans la zone extérieure, une forte variation saisonnière tandis que la zone profonde reste beaucoup plus stable.

L'écosystème souterrain possède une double frontière: L'entrée de la cavité et la zone de transition zone extérieure - zone profonde. Il existe donc deux ecotones (au sens d'Odum, 1959). L'étude quantitative montre l'évolution saisonnière des communautés habitant les deux régions écologiques. Le remaniement faunique de la zone extérieure est important et constitue une caractéristique de l'écosystème. Il est indispensable de tenir compte de ce phénomème pour comprendre l'évolution des biocénoses concernées.

\section{RESUME}

L'évolution de la composition faunique d'une cavité souterraine de géométrie simple (Trou des Nutons, Chaudfontaine, Belgique) a été étudiée. Des comparaisons quantitatives ont été effectuées entre les deux régions importantes de la caverne la zone extérieure et la zone profonde - en utilisant les coefficients de diversité et de similarité. Il apparaît que la zone extérieure est le siège d'un remaniement faunique important tandis que la zone profonde reste beaucoup plus stable. L'ensemble de la communauté cavernicole reste cependant relativement constant en fonction du temps. 


\section{SUMMARY}

The evolution of faunal composition in a subterranean cavity of simple structure (Trou des Nutons, Chaudfontaine, Belgium) has been studied. Quantitative comparisons have been carried on in the two most important parts of the cave - the external zone and the deep zone - using diversity and similarity coefficients. It has been found that the external zone presents a great redistribution of the fauna while the deep zone is more stable. However, the whole cave community stays relatively constant during time.

\section{BIBLIOGRAPHIE}

BRILLOUIN, L., 1960 - Science and information theory, Academic Press Inc., New-York.

CULVER, D.C. et POULSON, T.L., 1970 - Community boundaries: faunal diversity around a cave entrance.Ann. Spéléo., 25, 853-860.

HORN, H., 1966 - Measurement of overlap in comparative ecological studies, American Naturalist, 100, 419-424.

IVES, J.D., 1964 - Cave migration of certain insects. Nat. Speleo. Soc. Bull., 26, 115-118.

JEQUIER, J.P., 1964 - Etude écologique et statistique de la faune terrestre d'une caverne du Jura suisse au cours d'une année d'observation. Rev. suisse Zool., 71, 313-370.

MAC ARTHUR, R.H., 1965 - Patterns of species diversity. Biol. Rev., 40, 510-533.

MARGALEF, D.R., 1963 - On certain unifying principles in ecology. Amer. Naturalist, 97, 357-374.

MOTAS, C., DECOU, V., BURGHELE, A., 1967 - Sur l'association pariétale des grottes d'Olténie (Roumanie). Ann. Spéléo., 22, 475-522.

ODUM, E.P., 1959 - Fundamentals of Ecology, Saunders Co. ed., Philadelphia.

PIELOU, E.C., 1966 - The measurements of diversity in different types of biological collections. J. Theor. Biol., 13, 131-144.

PIELOU, E.C., 1967 - The use of information theory in the study of the diversity of biological populations, Proc. V. Berkeley Symposium on Mathematical Statistics and Probability, 4, 163-177.

POULSON, T.L. et CULVER, D.C., 1969 - Diversity in terrestrial cave communities. Ecology, 50, 153-158. 